heitlichung der Kommunalwahlen erschweren. Solange diese lokalen Besonderheiten zugelassen werden und institutionell abgesichert sind - zum Beispiel durch den Wegfall der Fünfprozentklausel bei Kommunalwahlen oder das stärker personalisierte Wahlrecht -, ist von einer bundespolitischen Durchdringung der Kommunalwahlen nicht zwangsweise auszugehen.

Die Nachteile einer horizontalen Vereinheitlichung der Kommunalwahlen sind damit nicht so stark zu gewichten wie im Fall einer Vereinheitlichung der Landtagswahlen. Umgekehrt könnten die Vorteile einer stärkeren bundesweiten Aufmerksamkeit genutzt werden. Sie kämen nicht nur der Wahlbeteiligung zugute. Vielmehr bekäme die Kommunalpolitik als dritte Ebene im politischen Mehrebenensystem insgesamt mehr Bedeutung, als ihr heute zuteil wird. Die Kommunen werden von den Politikern in Bund und Ländern, den Medien, aber auch von den Bürgern kaum wahrgenommen. Dies erstaunt angesichts der Tatsache, dass in der Bundesrepublik Deutschland die kommunale Selbstverwaltung nicht nur in Art. 28 Abs. 2 GG explizit garantiert ist. Vielmehr sind die Kommunen verantwortlich für die öffentliche Daseinsvorsorge, für die Bereitstellung und Versorgung der Bürger mit zentralen Gütern des täglichen Lebens und Zusammenlebens (Wasser- und Energieversorgung, Umweltschutz, Wirtschaftsförderung, Bauvorhaben, Ausländerintegration, Kinderbetreuung, sozialer Wohnungsbau etc.). Etwa ein Viertel aller Staatsausgaben werden über die örtlichen Verwaltungen getätigt, was die Bedeutung der Kommunen im gesamten Staatsgefüge verdeutlicht. ${ }^{31}$ Mehr Aufmerksamkeit der Politik und der Medien für die Kommunen wäre damit nicht nur vor dem Hintergrund einer höheren Wahlbeteiligung wünschenswert. Innerhalb des bundesdeutschen Mehrebenensystems mit seiner zu Unrecht fast ausschließlichen Fokussierung auf die Bundes- und Landespolitik käme sie einer stärkeren Anerkennung der lokalen Politik für das tägliche Lebensumfeld der Bürger gleich.

31 Vgl. Gerd Schmidt-Eichstaedt, Autonomie und Regelung von oben. Zum Verhältnis von kommunaler Eigenverantwortung und fremdbestimmter Eingrenzung durch Bundes- und Landesrecht sowie durch Normen der Europäischen Union, in: Hellmut Wollmann / Roland Roth (Hrsg.), Kommunalpolitik. Politisches Handeln in den Gemeinden, Opladen 1999, S. 323 337; Angelika Vetter, Changes in German Local Autonomy, in: Edward Page / Michael Goldsmith (Hrsg.), Local Autonomy in Europe (im Erscheinen).

\title{
Verfassungswandel in Großbritannien unter Labour - veränderte Handlungsspielräume des Parlaments
}

\author{
Merten Haring
}

Am 1. Mai 1997 gelang es Labour unter Tony Blair, die Wahlen zum Unterhaus zu gewinnen, eine Mehrheit von 118 Sitzen zu erzielen und dadurch mit 448 Abgeordneten die größte Unterhausfraktion in der Geschichte der Partei zu bilden und die Konservativen nach 18 Jahren als Regierungspartei abzulösen. Blair blieb, wie noch kein Labour-Chef vor ihm, drei Wahlen hintereinander ungeschlagen und amtierte zehn Jahre lang als Premierminister - auch dies ein Rekord unter Labour-Amtsinhabern. In seine Regierungszeit fallen 
Veränderungen der ungeschriebenen - genauer: nicht kodifizierten - Verfassung Großbritanniens ${ }^{1}$, die in ihrer Anzahl und Reichweite in der britischen Geschichte seit der Vereinigung von England und Schottland 1707 ohne Beispiel sind. ${ }^{2}$

Die britische Verfassung ist nicht in einem einzelnen Dokument höheren Rechts zusammengefasst, sondern speist sich aus verschiedenen Quellen: common law, statute law ${ }^{3}$, Vorrechte der Krone (die in der Praxis durch den Premierminister wahrgenommen werden) und wichtige wissenschaftliche Analysen der Verfassungsrealität (zum Beispiel die Arbeiten von Walter Bagehot). Weitere Quellen sind Verfassungskonventionen, die auf Traditionen, ungeschriebenen Verhaltensregeln sowie einem informellen Konsens gründen, von der Akzeptanz der Akteure abhängen ${ }^{4}$ und für das Funktionieren der Verfassung unerlässlich sind; hinzu kommt seit Neuestem die Europäische Union durch die Europäischen Verträge, Verordnungen und Richtlinien, den Europäischen Gerichtshof und die Grundrechtecharta.

Die zwei zentralen Prinzipien der britischen Verfassung sind die „Rule of Law“ und die uneingeschränkte Parlamentssouveränität. Die „Rule of Law“ schützt die Bürger vor staatlicher Willkür und bindet staatliches Handeln an Gesetze. Ohne ein legitimierendes Gesetz darf der Staat nicht aktiv werden. Das Prinzip der uneingeschränkten Parlamentssouveränität besagt, dass das Parlament und nicht das Volk Träger der Souveränität ist. Alle Macht geht vom Parlament beziehungsweise dem Unterhaus aus. Somit können nach britischem Staatsverständnis keine Verfassung und kein Gericht über den Gesetzen des Unterhauses stehen. Es kann mit einfacher Mehrheit das Oberhaus abschaffen, das diese Entscheidung durch sein Veto nur verzögern, aber nicht verhindern könnte. Auch könnte das Unterhaus auf der Basis dieser Souveränitätsdoktrin mit einfacher Mehrheit ein Todesurteil für die Königin beschließen - und sie müsste es unterschreiben. Wie im Zweiten Weltkrieg im Konsens aller Parteien geschehen, kann das Unterhaus, ebenfalls mit einfacher Mehrheit, seine eigene Wahl verschieben oder ganz aussetzen. Weder existiert ein Oberstes Gericht, das angerufen werden könnte, noch gibt es höheres Recht, an dem das Handeln des Unterhauses gemessen werden könnte. Obwohl also die Normen der nicht kodifizierten Verfassung formal jederzeit mit einfacher Mehrheit geändert werden könnten, passiert dies nicht einfach.

Die Parlamentssouveränität darf nicht zum Nennwert genommen werden, sondern bedarf einer Präzisierung im Lichte der Entwicklungen des britischen Regierungssystems. Die Mitglieder des Unterhauses werden in Ein-Mann-Wahlkreisen mit einfachem Mehrheits-

1 United Kingdom of Great Britain and Northern Ireland; im Folgenden vereinfachend Großbritannien.

2 André Kaiser führt für die Zeit nach 1945 nicht weniger als elf institutionelle Reformen auf, von denen nur zwei $(1958,1979)$ nicht unter Tony Blair durchgeführt worden sind; vgl. André Kaiser, Mehrheitsdemokratie und Institutionenreform, Frankfurt am Main 2002.

3 Dazu zählen wichtige Gesetze aus der Verfassungsgeschichte Großbritanniens, insbesondere die „Magna Charta“ von 1215, der „Habeas Corpus Act“ von 1679 und die „Bill of Rights" von 1689, der „Act of Union“ zur Vereinigung von England und Schottland von 1707 sowie die Gesetze zum Wahlrecht und zur Stellung des Oberhauses.

4 Vgl. ausführlich auch: Bernd Becker, Politik in Großbritannien, Paderborn 2002, S. 25 f; Rodney Brazier, The Constitution of the United Kingdom, in: Cambridge Law Journal, 58. Jg. (1999), H. 1, S. 96 - 128, S. 121 f.; Emil Hübner / Ursula Münch, Das politische System Großbritanniens. Eine Einführung, München 1999, S. 132 f. 
wahlrecht gewählt. Eine parlamentarische Mehrheit ist im Normalfall nicht Ergebnis der Wählerentscheidung, sondern wird vom Wahlsystem erzeugt. ${ }^{5}$ Der Premierminister wird von der größten Partei gestellt, die in der Regel über die absolute Mehrheit der Sitze verfügt; er kann mit einer in Demokratien einmaligen Machtfülle ohne Einschränkungen durch Opposition, Zweite Kammer oder ein Verfassungsgericht regieren. „Ein englischer Premierminister, der seiner Majorität im Parlament sicher ist, kann tun, was der deutsche Kaiser und der amerikanische Präsident und alle Komiteevorsitzende im Kongress der Vereinigten Staaten nicht tun können; denn er kann die Gesetze ändern, er kann Steuern auferlegen und aufheben, und er kann alle Staatsgewalten dirigieren. "6 Ein britischer Premierminister gerät nur dann in Gefahr, wenn er seinen Abgeordneten nicht mehr die nächste Wiederwahl garantieren kann. ${ }^{7}$ Aufgrund der Einheit der Parlamentsmehrheit im britischem Unterhaus und der von ihr getragenen Regierung sowie der Dominanz der Exekutive bedeutet jede Veränderung des Handlungsspielraums des Parlaments ${ }^{8}$ durch Verfassungswandel eine Änderung des Handlungsspielraums der Regierung - und umgekehrt. ${ }^{9}$

\section{Verfassungswandel}

Aufgrund der Natur des britischen Regierungssystems und seiner Verfassung kommt dem Verfassungskonsens und den ungeschriebenen Konventionen eine erhebliche Bedeutung zu. Da es formal an jeglicher Beschränkung der Herrschaft der einfachen Mehrheit im Unterhaus durch andere Institutionen fehlt und die einzige Kontrolle durch den Wähler stattfindet, muss eine breite Zustimmung zur Verfassung vorhanden sein, um die Entscheidungen der Mehrheit für die Minderheit akzeptabel zu machen.

In Großbritannien waren es in den letzten Jahrzehnten verschiedene Faktoren, die gemeinsam die Legitimität des Regierungssystems und den Verfassungskonsens in Frage stell-

5 Als Extremfall kann die Unterhauswahl von 1983 gelten; dabei gewann die Liberale Partei in einer Allianz mit der Sozialdemokratischen Partei 25,4 Prozent der Stimmen, konnte aber aufgrund des Wahlrechtes nur 23 Sitze im Unterhaus gewinnen. Labour errang 27,6 Prozent der Stimmen und konnte 209 Abgeordnete entsenden. Die Konservativen brauchten damit für einen Sitz im Unterhaus 33.000 Stimmen, Labour ungefähr 7500 mehr und die Allianz unglaubliche 338.000 Stimmen.

6 Sidney Low, Die Regierung Englands, Tübingen 1908, S. 45.

7 Es waren letztendlich auch die konservativen Unterhausabgeordneten und nicht die Wähler, die Margaret Thatcher nach elf Jahren als Premierministerin aufgrund ihrer Politik der Poll-Tax stürzten, da sie ihre Chance auf eine Wiederwahl nicht mehr gewahrt sahen.

8 Obwohl es sich in Großbritannien formal um ein Parlament mit zwei Kammern handelt, ist es real ein Einkammerparlament. Reformen in den Jahren 1911 und 1949 haben dazu geführt, dass das Oberhaus Gesetze nur noch mittels eines aufschiebenden Vetos für ein Jahr aufhalten kann. Bei Finanzgesetzen beträgt die Frist zwei Monate. Der Premierminister selber ist nur dem Unterhaus gegenüber verantwortlich.

9 Vgl. zum Verhältnis von Exekutive und Legislative ausführlich auch: Arend Lijphart, Patterns of Democracy. Government Forms und Performance in Thirty-Six Countries, New Haven 1999, S. 10, S. 28; Stefan Fröhlich, Vom „Prime Ministerial Government“ zur „British Presidency“?, in: APuZ, B 18 (1997), S. 31 - 37, S. 31; Emil Hübner / Ursula Münch, a.a.O. (Fn. 4), S. 126 f., 141 f.; Bernd Becker, a.a.O. (Fn. 4), S. 124. 
ten. ${ }^{10}$ Bis zur Amtszeit von Margaret Thatcher herrschte ein verfassungspolitischer Konsens zwischen allen maßgeblichen gesellschaftlichen und politischen Gruppen. Sie nutzte den ihr als Premierministerin zustehenden Handlungsspielraum allerdings rücksichtslos aus und ging zum Beispiel sehr energisch gegen Gewerkschaften und die kommunale Ebene vor. Ihre Politik befand sich in einer doppelten Legitimationskrise: Auf der einen Seite wurde erhebliche Kritik daran geübt, dass die Konservativen bei ihren Wahlsiegen 1979 bis 1992 nie mehr als 43,9 Prozent aller Stimmen bekamen, aber unkontrolliert und vor allem radikal alle Machtinstrumente der Mehrheitspartei nutzten. Daneben wurden auch die immer stärker werdenden Verzerrungen des Wahlsystems Gegenstand der Verfassungskritik. Im Laufe der Regierungszeit der Konservativen von 1979 bis 1997 kam es zu einer wahlgeographischen Spaltung Großbritanniens. In Schottland, Wales und im englischen Norden bildeten sich Hochburgen von Labour, während die Konservativen vor allem im Süden ihre Stimmen gewannen. Da der Süden Englands aber erheblich dichter bevölkert war, reichte dies für eine Mehrheit im Unterhaus aus. Somit wurden geographisch große Teile des Landes von einer Mehrheit der englischen Unterhausabgeordneten regiert, die gleichzeitig die kommunale Selbstverwaltung immer mehr beschnitt. „It has been increasingly argued that the steady decline of electoral support for the Conservative party, combined with a direction of central government policy that conflicts with what was widely seen to be the interests of people living in Scotland, has created a sense of political alienation drawing heavily on feelings of national distinctiveness. " ${ }^{11}$ Die Bürger bekamen den Eindruck, hilf- und grenzenlos den Zugriffen einer Regierung ausgesetzt zu sein, deren Legitimität angezweifelt wurde. Die Gewaltenteilung, auf die das Westminster-Modell ausdrücklich verzichtet, wurde nun gefordert, um ein Gegengewicht zur Regierung zu bilden.

Thatchers Sturz durch die eigene Partei zeigte aber in den Augen der Verteidiger der Verfassung, dass selbst ein so mächtiger Premier wie sie Grenzen habe. Als Katalysator wirkten dann allerdings die Wahlen von 1992. Allen Erwartungen zum Trotz hatten es John Major und die Konservativen geschafft, erneut den Sieg davonzutragen. Große Kreise der britischen Öffentlichkeit sahen damit einen der wichtigsten Bestandteile des Westminster-Modells, die alternierenden Regierungen, gefährdet. Aufgrund des Fehlens einer Gewaltenteilung stellt die Kontrolle durch den Wähler den entscheidenden Aspekt des britischen Regierungssystems dar. Da aber Labour trotz der Wirtschaftskrise und des schlechten Auftretens der Konservativen die Wahl - entgegen allen Meinungsumfragen - nicht gewonnen hatte, wurde nun intensiv diskutiert, ob sich in Großbritannien eine politische Konstellation ergeben hätte, die zu einer strukturellen relativen Mehrheit der Konservativen führte und somit aufgrund des Wahlsystems deren Regierung dauerhaft zementieren würde.

Schon 1988 war mit „Charter 88“ eine Initiative gegründet worden, die die zahlreichen überparteilichen Initiativen zur Reform der britischen Verfassung bündelte. Sie erhielt nach der Wahl 1992 immer mehr Zulauf aus allen Bevölkerungsschichten und übte massiven

10 Vgl. ausführlich Merten Haring, Verfassungswandel in Großbritannien. Von Margaret Thatcher bis Tony Blair, Osnabrück 2006; Hans Kastendiek, Traditionelles und neues Verfassungsdenken in Großbritannien, in: Gert-Joachim Glaeßner / Werner Reutter / Charlie Jeffery (Hrsg.), Verfassungspolitik und Verfassungswandel in Deutschland und Großbritannien im Vergleich, Wiesbaden 2001.

11 Rodney Barker, Legitimacy in the United Kingdom: Scotland and the Poll Tax, in: British Journal of Political Science, 22. Jg. (1992), H. 4, S. $521-533$. 
Druck auf die britischen Parteien aus. Die Konservativen sprachen sich gegen jegliche Reform aus, und die Liberalen traten für eine weitgehende Revision ein, schürten damit allerdings auch Ängste in der Bevölkerung. Labour unter Tony Blair nahm 1997 eine mittlere Position ein: Im Unterschied zu 1992 zeigte die Partei klar Grenzen und Möglichkeiten von Verfassungsreformen auf und stellte sich mit ihren Forderungen zwischen Konservative und Liberale. Eine Folge dieser Neuorientierung war, dass die Reformvorschläge nicht ein geschlossenes Konzept ergaben, sondern abhängig vom Themengebiet eine mehr oder minder große Reichweite hatten. Sie unterschieden sich von „normalen“ politischen Vorhaben und Reformen dadurch, dass mit ihnen Elemente der Verfassung geändert werden sollten, die aus aktuellen Anlässen in den politischen Streit geraten waren, aber die seit Jahrhunderten unveränderte und akzeptierte Grundstruktur und das geprägte Verständnis des Regierungssystems betrafen.

\subsection{Devolution}

Eines der Kernstücke der umfangreichen Verfassungsreformen Labours stellt die Politik der Devolution dar. Dabei werden Kompetenzen des Zentralstaates, also im Endeffekt Kompetenzen der Mehrheit im Unterhaus und der von ihr getragenen britischen Regierung, auf gewählte Körperschaften und deren Exekutiven in einzelnen Landesteilen Großbritanniens übertragen. Der Umfang der Devolution suchte seinesgleichen: „... the Parliament of the United Kingdom will have pushed out from London to Belfast, Edinburgh and Cardiff constitutional autonomies not seen in those capitals for 200 years and more." 12 Diese „Dezentralisierung politischer Handlungsbefugnisse"13, die vor allem in Schottland gefordert wurde, stellte einen wichtigen Themenkomplex der britischen Innenpolitik dar, der innerhalb einer breiten Öffentlichkeit sehr intensiv diskutiert und beobachtet wurde. Er kann ohne weiteres als populärstes und für die Wähler wichtigstes Vorhaben der von Labour auf den Weg gebrachten Verfassungsreformen bezeichnet werden. Die Devolution hatte dabei in jeder Region des Vereinigten Königreichs eine spezielle Reichweite und Bedeutung. ${ }^{14}$ Gemein ist ihnen, dass aufgrund der uneingeschränkten Parlamentssouveränität rein formal jedwede Stufe der Devolution jederzeit vom britischen Unterhaus mit einfacher Mehrheit aufgehoben werden kann. ${ }^{15}$ Legitimität für die Devolution erwächst formell nicht aus dem Volk, sondern aus einem Gesetz des Unterhauses.

Schottland: Bereits knapp zwei Monate nach dem Wahlsieg von Labour im Mai 1997 stellten die jeweiligen Regionalministerien von Schottland und Wales in zwei Weißbüchern Konzepte für die Übertragung von Gesetzgebungskompetenzen vor, und die Gesetze über die Durchführung von Referenden über die Politik der Devolution wurden im Unterhaus verabschiedet. Am 11. September 1997 stimmte die schottische Bevölkerung in einem

12 Rodney Brazier, a.a.O. (Fn. 4), S. 115.

13 Bernd Becker, a.a.O. (Fn. 4), S. 63.

14 Die hier notwendigerweise knappe Skizze dieser Entwicklung schließt den besonders komplexen und komplizierten Verlauf der Devolution in Nord-Irland nicht ein.

15 Dass es sich hierbei nicht um ein Gedankenspiel handelt, zeigt die Auflösung des nordirischen Parlaments und damit die Rücknahme der Devolution 1972. 
Referendum über zwei Fragen ab: „Will Schottland ein Parlament?“ und „Will Schottland ein Parlament mit dem Recht, Steuersätze zu ändern?" Eine Koalition von Labour, den Liberaldemokraten und schottischen Nationalparteien rief zur Zustimmung auf, während sich die konservative Partei für ein „Nein“ aussprach. Bei einer Wahlbeteiligung von 60,2 Prozent stimmten bei der ersten Frage 74,3 Prozent und bei der zweiten Frage 63,5 Prozent mit "Ja“. Unverzüglich wurde in das Unterhaus ein Gesetzentwurf eingebracht, und im November 1998 trat der "Scotland Act“ in Kraft. Das schottische Parlament hat die alleinige Gesetzgebungskompetenz in folgenden Bereichen: Gesundheits-, Erziehungs- und Bildungspolitik, Kommunalverwaltung, Wohnungsbau, wirtschaftliche Entwicklung, Verkehr, Umwelt, Landwirtschaft, Sport, Kultur, Tourismus, soziale Dienste, Polizei sowie materielles und formelles Zivil- und Strafrecht. Beim Unterhaus verbleibt die Gesetzgebungskompetenz in der Außen-, Verteidigungs- und Sicherheitspolitik sowie der Finanz-, Arbeitsund Sozialpolitik. Neben den Kompetenzen im Bereich der Gesetzgebung verfügt das schottische Parlament noch über das Recht, den Basissatz der Einkommensteuer um maximal drei Prozent zu erhöhen oder zu senken. ${ }^{16}$ Abgesehen davon wird der Haushalt von den Zuweisungen der Zentralregierung in London getragen. Das Parlament selber wird in einer Kombination von Mehrheits- und Verhältniswahlrecht gewählt, das Koalitionen notwendig macht. ${ }^{17}$

Wales: Die Reichweite der Devolution in Wales ist bei Weitem nicht so groß wie in Schottland. Während sich aufgrund der Bestimmungen des Unionsvertrages von 1707 ein eigenes Rechtssystem und eine eigene Kirche erhalten haben, der Nationalismus und die Abgrenzung gegenüber England eine breite Öffentlichkeit erfassten, stand in Wales vor allem die Forderung nach dem Erhalt der kulturellen Identität und der eigenen Sprache im Vordergrund. Am 18. September 1997 fand in Wales ein Referendum über die Devolution statt. Abgestimmt wurde über die Bildung einer eigenen Versammlung, der „Welsh Assembly“, die über erheblich weniger Kompetenzen als das schottische Parlament verfügen sollte. Damit wurde bereits auf die geringeren Dezentralisierungsforderungen durch die Waliser selbst reagiert. Am Referendum, gegen das sich wieder vor allem die Konservativen stellten, beteiligten sich nur 50,1 Prozent der Wahlberechtigten. 50,3 Prozent stimmten der Einrichtung einer Versammlung zu, und 49,7 Prozent lehnten dies ab. Diese geringe Zustimmung spiegelte sich auch geographisch wider. Alle westlichen Stimmbezirke sprachen sich für die Devolution aus, während alle an England grenzenden dagegen votierten. Mit dem „Government of Wales Act“ im Juli 1998 wurde die „Welsh Assembly“ eingerichtet, die über keine eigene Gesetzgebungsbefugnis verfügt. Sie hat lediglich Vollzugskompetenzen

16 Aufgrund dieser beschränkten Kompetenzen machte sich bald Ernüchterung breit und das schottische Parlament beschäftigte sich intensiv mit dem Neubau eines Parlamentsgebäudes, das mit über 400 Millionen Pfund zehnmal so teuer wurde wie geplant. Dennoch wurden bauliche Mängel offensichtlich, als sich im März 2006 ein Dachträger löste und beinahe die englandfreundliche konservative Fraktion erschlagen hätte.

17 Vgl. Bernd Becker, a.a.O. (Fn. 4), S. 65 f.; Marc-Oliver Pahl, Das Vereinigte Königreich - Der Fortgang des Devolution-Prozesses und die Mitwirkung der Regionalinstitutionen im Bereich der Europäischen Union, in: Europäisches Zentrum für Föderalismus-Forschung Tübingen (Hrsg.), Jahrbuch des Föderalismus 2001, S. 281 - 295, S. 282 f.; The Constitution Unit, http://www. ucl.ac.uk/constitution-unit/publications/constitutional-update/devolution.html (Abruf am 13. November 2009); Emil Hübner / Ursula Münch, a.a.O. (Fn. 4), S. 58 f. 
und in einigen wenigen Bereichen das Recht zu konkretisierenden Ausführungsgesetzen. Eine eigene Steuerhoheit existiert ebenfalls nicht. Allerdings darf die Versammlung in ihrer Eigenschaft als ausführendes Organ über die Verwendung der Haushaltsmittel entscheiden, die zuvor dem Ministerium für Wales in London unterstanden. ${ }^{18}$

England: In England mit seinen 83 Prozent der Gesamtbevölkerung Großbritanniens findet sich kein Nationalismus, der mit jenem in Wales, Schottland oder Nordirland vergleichbar wäre. Konsequenterweise gibt es kaum Forderungen nach einer starken Dezentralisierung. Ungeachtet dessen zog die Devolution in den anderen Landesteilen auch Veränderungen in England selbst nach sich. Mit dem „Regional Development Agencies Act“ von 1998 wurde auch die Gründung von „Regional Assemblies“ in Aussicht gestellt. Bis Mitte 1999 hatten sich in allen acht Regionen „Voluntary Regional Chambers" etabliert. Sie bestehen zu 70 Prozent aus Amtsträgern, die in ihrer jeweiligen Kommune für ein Amt gewählt worden sind und zu 30 Prozent aus Vertretern von Interessengruppen wie Unternehmern, Gewerkschaftern, Umweltorganisationen oder Vertretern von Bildungseinrichtungen. Über die realen Kompetenzen und Aufgabenfelder herrscht auch unter den Beteiligten eher Unklarheit. Die meisten regionalen Kammern haben sich in „Regional Assemblies“ umbenannt, was aber nichts daran ändert, dass die Zusammensetzung nicht auf direkte Wahlen zurückgeht. Am 8. Mai 2003 wurde der „Regional Assemblies (Preparations) Act“ verabschiedet. Das „Boundary Committee for England“ wurde beauftragt, Vorschläge zu erarbeiten, wie gewählte Regionalversammlungen in England gestaltet werden könnten und legte am 1. Dezember 2003 seinen Bericht vor. Im Juni 2003 avisierte die Regierung von Tony Blair ein Referendum für den 4. November 2004, mit dem die Einwohner der drei nordöstlichen Regionen Englands die Möglichkeit erhielten, über die Einführung gewählter Regionalvertretungen zu entscheiden. Diese hätten am 1. April 2006 ihre Arbeit aufnehmen sollen. Der 1. April, auch „April Fools’ Day“ genannt, ist der traditionelle Tag, an dem Kommunalreformen in Großbritannien in Kraft treten. Das Ergebnis der Abstimmung war eindeutig: Nur 22 Prozent stimmten für die Einführung, 78 Prozent dagegen. „Following ... the referendum ..., Labour was quick to distance itself from the concept of elected regional assemblies. "19 Eine weitergehende Regionalisierung Englands war mit diesem Ergebnis, das in seiner Eindeutigkeit von keiner Partei angezweifelt wurde, an ihr vorläufiges Ende gekommen. ${ }^{20}$

18 Vgl. Bernd Becker, a.a.O. (Fn. 4), S. 69 - 71; Marc-Oliver Pahl, a.a.O. (Fn. 17), S. 284 f.; Rosanne Palmer / Charlie Jeffery, Das Vereinigte Königreich: Die „Devolution-Revolution“ setzt sich fort, in: Europäisches Zentrum für Föderalismus-Forschung Tübingen (Hrsg.), Jahrbuch des Föderalismus 2002, S. 349 - 351; The Constitution Unit, http://www.ucl.ac.uk/constitution-unit/publications/constitutional-update/devolution.html (Abruf am 13. November 2009).

19 The Constitution Unit, http://www.ucl.ac.uk/constitution-unit/publications/constitutionalupdate/english-regions.html (Abruf am 13. November 2009).

20 Vgl. Bernd Becker, a.a.O. (Fn. 4), S. 71 - 75; Marc-Oliver Pahl, a.a.O. (Fn. 17), S. 284 f.; The Constitution Unit, http://www.ucl.ac.uk/constitution-unit/publications/constitutional-update/ devolution.html und http://www.ucl.ac.uk/constitution-unit/publications/constitutional-update/ english-regions.html (Abruf am 13. November 2009); Emil Hübner / Ursula Münch, a.a.O. (Fn. 5), S. 62 - 64; Rodney Brazier, a.a.O. (Fn. 5), S. 116 f.; Jonathan Bradbury, The political dynamics of sub-state regionalism, in: British Journal of Politics and International Relations, 5. Jg. (2003), H. 4, S. $543-575$, S. 567. 


\subsection{Kommunalreformen}

Nach der Wahl 1997 begann eine breite Debatte über eine Reform der lokalen Ebene. Neben den bereits angesprochenen Veränderungen durch die Devolution, die sich bis auf die kommunale Verwaltung erstrecken, wurde nun auch die unterste Ebene von konkreten Programmen erfasst. Die Kommunalverwaltung sollte bürgernäher, effektiver und demokratischer organisiert werden. 1998 wurden die Ideen in einem Weißbuch „Modern Local Government: In touch with the people“ zusammengefasst. Reformen sollten vor allem durch verstärkte Partizipationsmöglichkeiten der Einwohner mittels „Local Forums“ und durch Veränderungen bei den Wahlbestimmungen erreicht werden. Mit den „Local Government Acts“von 1999 und 2000 wurde die Führung der Kommunen neu geregelt. Eine der Reformen umfasste die Möglichkeit der Direktwahl des Bürgermeisters, über deren Anwendung die Bürger in Abstimmungen entscheiden konnten. Davon machten 22 Gemeinden Gebrauch, von denen 15 gegen die Direktwahl und nur sieben dafür stimmten. Drei dieser Bürgermeisterwahlen konnte Labour für sich entscheiden und zwei die Liberalen. In Hartlepool gewann mit Stuart Drummond ein unabhängiger Kandidat die Wahl, der im Wahlkampf als Affe verkleidet im Kostüm des Maskottchens des örtlichen Fußballclubs und mit dem Slogan „free bananas for schoolchildren“ aufgetreten war. Unter dem Namen „H’Angus the Monkey“ setzte er sich gegen die anderen Kandidaten durch und wurde erster direkt gewählter Bürgermeister (mit einem Jahreseinkommen von 53.000 Pfund). Auch das Ergebnis in London trug aus Sicht von Labour nicht zu einem Erfolg des Projektes bei: „War dieses Vorhaben in der ersten Legislaturperiode noch eines der zentralen Projekte der Reformpolitik New Labours, ist der Enthusiasmus spätestens seit der Wahl Livingstones in London innerhalb der Regierung deutlich abgeflaut. "21 Da die Resultate also mager ausfielen und durch Hartlepool die ganze Idee direkt gewählter Bürgermeister in Frage gestellt wurde, fand die Reform in der Folge kaum noch größere Beachtung. Ein Erfolg wurde dagegen die Erprobung von Pilotprojekten wie Briefwahl oder Internetwahl, durch die die Beteiligung erheblich anstieg. Die Regierung kündigte daraufhin an, in $\mathrm{Zu}-$ kunft die Möglichkeiten zur Briefwahl ausbauen zu wollen. ${ }^{22}$

Von besonderer Bedeutung innerhalb der Kommunalreformen sind die Veränderungen in London. 1985 hatte Margaret Thatcher den „Greater London Council“ abgeschafft, weil sie ihn aufgrund seiner starken linken Labour-Prägung als Gegner und Ärgernis ansah. So brachte der Bürgermeister von London zum Beispiel in Sichtweite des Unterhauses eine große Anzeigetafel mit den aktuellen Arbeitslosenzahlen Großbritanniens an. Dank der uneingeschränkten Parlamentssouveränität war es für Thatcher rein rechtlich kein Problem, die Selbstverwaltung innerhalb weniger Tage zu beenden. In Reaktion auf diese in der Öffentlichkeit unpopuläre Maßnahme, die London zur einzigen Großstadt der westlichen Welt ohne eigene Kommunalverwaltung machte, versprach Labour im Wahlkampf 1997 ein Referendum über die Wiedereinrichtung einer Selbstverwaltung. Am 7. Mai 1998 stimmten bei einer Beteiligung von lediglich 34 Prozent der Wahlberechtigten 72 Prozent für die Di-

21 Bernd Becker, a.a.O. (Fn. 4), S. 102.

22 Vgl. ebenda, S. 100 - 103; Michael Cole, Local Government Reform in Britain 1997-2001, in: Government and Opposition, 38. Jg. (2003), H. 2, S. 108 - 202; The Constitution Unit, http:// www.ucl.ac.uk/constitution-unit/publications/constitutional-update/elections-and-parties.html (Abruf am 13. November 2009). 
rektwahl eines Bürgermeisters und die Einführung einer gewählten Versammlung mit 25 Mitgliedern. Nach der Nominierung der Spitzenkandidaten, die für Konservative und Labour jeweils äußerst kontrovers verlief und ihr Image stark beschädigte, wurde am 5. Mai 2000 Ken Livingstone („Red Ken“) als unabhängiger Kandidat zum Bürgermeister gewählt.

\subsection{Reform der Zentralbank}

Völlig im Schatten der Devolution stehend, ist die Reform der Zentralbank gleichwohl eine der bedeutendsten Veränderungen der britischen Verfassung innerhalb des Bündels der Reformen nach 1997, auch wenn sie von der Öffentlichkeit am wenigsten wahrgenommen wurde. „Ein entscheidender Faktor der Erfolgsgeschichte der Wirtschaftspolitik New Labours ist die Entlassung der Bank von England in die Unabhängigkeit gleich nach dem Wahlsieg 1997. "23 War die Bank of England bisher den Eingriffen der Regierung und deren politischen Wünschen vollständig ausgeliefert, sollte sich dies nun ändern. „Hierzu war bisher noch keine ... britische Regierung bereit gewesen. "24 Die Regierung Blair beugte sich damit dem Druck der Wirtschaft, folgte den Vorgaben der EU und konnte zugleich die die Regierung durchaus auch belastenden geldpolitischen Zuständigkeiten an eine unabhängige Institution abgeben. Innerhalb von lediglich vier Tagen nach Amtsantritt der neuen Labour-Regierung wurde die Bank of England als Zentralbank in die weitgehende Unabhängigkeit entlassen. Das Finanzministerium übertrug ihr die Kontrolle über die Zinssätze und die Wahrung der Geldwertstabilität, womit die Regierung die Hoheit über die Geldpolitik freiwillig abgegeben hatte. Künftig können Regierungen also beispielsweise nicht mehr direkt vor Wahlen die Leitzinsen aus taktischen Gründern ändern oder die Regeln zur Zählung von Arbeitslosen den eigenen (wahl-)politischen Bedürfnissen anpassen.

\subsection{Reform des Oberhauses}

Das Oberhaus verfügt nur über sehr geringe Möglichkeiten, die Gesetzgebung der Mehrheitsfraktion im Unterhaus zu beeinflussen. Allerdings ist es teilweise in der Lage, ein Gesetz so an das Unterhaus zurückzuverweisen, dass es in der laufenden Sitzungsperiode nicht mehr beraten werden kann und somit verfällt. Aufgrund seiner Zusammensetzung spielt das Oberhaus für den Premierminister eine große Rolle. Seine Mitglieder rekrutierten sich bis 1958 aus erblichen Mitgliedern, den „Hereditary Peers“, sowie zu einem kleinen Teil aus Geistlichen und den „Law Lords“. 1958 erhielt der Premierminister das Recht, Mitglieder des Oberhauses auf Lebenszeit (Life Peers) zu ernennen und so bedeutenden Einfluss auf die Zweite Kammer zu gewinnen. „Das Instrument der Ernennung, das letztendlich vom Premierminister kontrolliert wird, erschafft ein ganzes Universum von Patronagemöglichkeiten ohne Mitwirkung des Parlaments und stärkt damit dessen Rolle in der Regierung. " 25 Neben der Möglichkeit, Gefolgsleute zu belohnen und an sich zu binden, bietet das Oberhaus für

23 Bernd Becker, a.a.O. (Fn. 4), S. 57.

24 Heidrun Abromeit, Entwicklungslinien im Verhältnis von Staat und Wirtschaft, in: Hans Kastendiek (Hrsg.), Länderbericht Großbritannien, Bonn 1998, S. 358 - 378, S. 368.

25 Roland Sturm, Großbritannien: Wirtschaft, Gesellschaft, Politik, Opladen 1997, S. 213. 
den Premierminister auch die Chance, politische Konkurrenten zu ernennen und sie - aufgrund der Unvereinbarkeit der Mitgliedschaft im Oberhaus mit vielen anderen Funktionen - in ihrer politischen Entfaltung zu beschränken und quasi „wegzuloben“.

Die erste Stufe der Reformen des Oberhauses durch die Blair-Regierung umfasste die Abschaffung der erblichen Sitze. Mit dem „House of Lords Act“ von 1999 wurde ihre Anzahl auf 92 verringert, womit die auf Lebenszeit vom Premierminister ernannten Mitglieder des Oberhauses mit mehr als 500 nun die größte Gruppe stellen. ${ }^{26}$ Im Februar 1999 wurde unter Lord Wakeham eine Kommission einberufen, die Vorschläge für eine umfassende Reform des Oberhauses erstellen sollte. Im Januar 2000 wurde der Bericht „A House for the Future" vorgelegt. Dieser fasste alle Kritik am Oberhaus zusammen, zeigte aber zugleich die mögliche Zukunft auf. Im Bericht wird die Aufgabe der Zweiten Kammer darin gesehen, Stimme aller Teile der britischen Gesellschaft, aller Nationen und Regionen Großbritanniens zu sein. Daneben sollte es in seiner Rolle als Kontroll- und Überprüfungsinstanz verstärkt werden. Die Reformen gerieten allerdings schnell ins Stocken, da trotz einer Vielzahl von Einzelvorschlägen insbesondere keine Einigkeit über die Bestellung der Mitglieder des Oberhauses erzielt werden konnte. Im Prinzip gibt es drei diskutierte Varianten: komplette Wahl (wobei sich die Frage des Wahlverfahrens stellt), teilweise Wahl und teilweise Ernennung sowie vollständige Ernennung der Mitglieder. Sicher ist derzeit, dass die Bevölkerung ein reformiertes Oberhaus und weder Abschaffung noch Stillstand möchte. Welchen Weg die Reformen nehmen werden, ist aber nicht abzusehen. „Anyone not equipped with a crystal ball in good working order must necessarily be cautious in making any prediction about the ultimate shape of a reformed House." 27

\subsection{Reform des Unterhauses}

Das Unterhaus wurde beim Amtsantritt von Tony Blair bei Weitem nicht mit dem Reformeifer bedacht, der sonst die Politik von Labour prägte. Das Regierungsprogramm für die erste Amtszeit von 1997 bis 2001 benannte keine konkreten Vorhaben, sondern beschränkte sich darauf, zu betonen, dass es Aufgabe des Parlamentes selbst und nicht der Regierung wäre, Vorschläge zu erarbeiten. Die Konservative und die Liberale Partei entwickelten im Unterschied dazu klare Vorstellungen zur Reform und zur Stärkung des Unterhauses, die sie jeweils im Jahr 2000 der Öffentlichkeit präsentierten. Ein Mangel an Reformvorschlägen existierte somit nicht. Hier zeigt sich der naturgemäße Gegensatz zwischen Regierung und Opposition. Überspitzt formuliert ist das Unterhaus für die Regierung und vor allem für den Premierminister dazu da, die Regierungspolitik zu legitimieren, jedoch nicht selbstständig Entscheidungen zu treffen oder Rechenschaft von der Regierung zu verlangen. Die jeweilige Opposition sieht dagegen gern die Rolle des Unterhauses gestärkt, um

26 Vgl. Bernd Becker, a.a.O. (Fn. 4), S. 114; House of Lords Information Office, Briefing Papers List: History of the House of Lords, http://www.parliament.uk/documents/upload/HofLBpHistory.pdf (Abruf am 13. November 2009); André Kaiser, a.a.O. (Fn. 2), S. 402 f.; The Constitution Unit, http://www.ucl.ac.uk/constitution-unit/publications/constitutional-update/hol-reform. html (Abruf am 13. November 2009).

27 Sir Bingham, Lord of Cornhill, A New Supreme Court for the United Kingdom, London 2002, S. 4. 
die Regierung stärker kontrollieren zu können. Nach dieser Sichtweise bestand für die Labour-Regierung kein Eigeninteresse an einer solchen Reform; verändert wurde schließlich nur die traditionelle Befragung des Premierministers: Sie findet nicht mehr zweimal die Woche jeweils 15 Minuten, sondern einmal die Woche 30 Minuten statt.

So dürfte sich wohl die in der Öffentlichkeit geübte Kritik an den schwachen Kontrollinstrumenten des Unterhauses und einer zu starken Dominanz der Exekutive fortsetzen, die schon seit Ende der 1960er Jahre erhoben wurde und sich in der Amtszeit von Margaret Thatcher verstärkte. Die wichtigsten der wenigen Kontrollinstrumente sind schriftliche und mündliche Anfragen, parlamentarische Anfragen und der Rechnungsprüfungsausschuss. 1979 wurden diese durch die Einrichtung von Select Committees ergänzt. Dabei handelt es sich um Ausschüsse, die jeweils die Arbeit eines Ministeriums kritisch begleiten und gemäß den Mehrheitsverhältnissen im Unterhaus besetzt sind. Allerdings, und darauf wurde sehr großes Gewicht gelegt, sind sie in keiner Weise in den Gesetzgebungsprozess eingebunden. Sie können Regierungsvertreter und Beamte lediglich um Auskunftserteilung bitten, aber weder zum Erscheinen noch zur Auskunft zwingen. ${ }^{28}$ Parteiübergreifend wurde vor der Gefahr von Fachausschüssen, die sich zum Beispiel nach deutschem Vorbild aus Experten der Fraktionen rekrutieren, sich in das Entscheidungsrecht der Exekutive einmischen und damit überparteiliche Koalitionen und fraktionsinterne Spannungen erzeugen könnten, gewarnt. Solche Ausschüsse werden als Widerspruch zum britischen Regierungssystem und seiner klaren Zuteilung politischer Verantwortung aufgefasst.

\subsection{Reform des Wahlrechts}

Das in Großbritannien angewandte relative Mehrheitswahlrecht verzerrt den Wählerwillen mit dem Ziel klarer und eindeutiger Mehrheiten im Parlament, wobei die beiden großen Parteien begünstigt werden. Parteien, die über eine breite Unterstützung verfügen, denen aber regionale Hochburgen fehlen, werden demgegenüber benachteiligt. Im Wahlprogramm von 1997 hatte Labour angekündigt, das schottische Parlament und die walisische Nationalversammlung nach einer Form der Verhältniswahl zu bestimmen. Zur Reform des Wahlrechts zum Unterhaus sollte eine unabhängige Kommission eingesetzt und so ein Vorschlag erarbeitet werden, über den die Bürger dann abstimmen sollten.

Am 6. Mai 1999 wurde das erste schottische Parlament seit der Union mit England 1707 gewählt. Dabei wurde im Gegensatz zu den nationalen Wahlen nicht das Mehrheitswahlrecht, sondern eine Kombination von Mehrheits- und Verhältniswahlrecht, das „Additional Member System“, angewendet. Der Wähler hat eine Stimme für den Wahlkreiskandidaten und eine für die Parteiliste. 73 der Abgeordneten (MSP, „Member of the Scottish Parliament") werden wie bisher in 73 Wahlkreisen nach dem relativen Mehrheitswahlrecht gewählt. Die restlichen 56 werden in den acht schottischen Europawahlkreisen nach dem Verhältniswahlrecht gewählt, wobei jeder Wahlkreis sieben Abgeordnete entsendet und die erreichten Direktmandate berücksichtigt werden. 1999 konnte Labour mit 39 Prozent der Wahlkreisstimmen 53 der Wahlkreise und mit 34 Prozent der Listenstimmen weitere drei Listenmandate, somit insgesamt 56 Sitze gewinnen. Die Konservativen hingegen erhielten

28 Vgl. Roland Sturm, a.a.O. (Fn. 25), S. 210 f.; Bernd Becker, a.a.O. (Fn. 4), S. 123. 
mit 15,6 Prozent der Wahlkreisstimmen zwar keinen einzigen Wahlkreis und wären nach dem Unterhaus-Wahlrecht nicht im Parlament vertreten, konnten aber über die Parteilisten mit 15,4 Prozent der Stimmen 18 Listenmandate erobern. Die Liberaldemokraten errangen 14,2 Prozent der Wahlkreisstimmen - also 1,4 Punkte weniger als die Konservativen -, konnten damit aber zwölf Wahlkreise erobern. Weitere fünf Sitze bekamen sie aufgrund von 12,5 Prozent der Listenstimmen. Deutlich wird hier, wie das neue Wahlrecht das Wahlergebnis verändert. Labour hätte, wenn man das Resultat der Wahlkreise zu Grunde legt, mit einer Zweidrittelmehrheit regiert, während die Partei nun in eine Koalition mit den Liberaldemokraten gehen musste. Ein ähnliches Bild findet sich bei der gleichzeitig stattgefundenen Wahl zur walisischen Nationalversammlung. Auch hier hätte Labour nach dem Unterhaus-Wahlrecht der reinen Mehrheitswahl 27 der Wahlkreise und damit eine Zweidrittelmehrheit der Wahlkreise gewonnen und befand sich nach Anwendung des neuen „Additional Member Systems“ wieder in der Situation, eine Koalition eingehen zu müssen. ${ }^{29}$

Bei den EP-Wahlen wird gemäß dem „European Parliamentary Elections Act“ von 1999 ebenfalls nicht das relative Mehrheitswahlrecht, sondern ein reines Verhältniswahlrecht angewandt. Dazu wurde Großbritannien in elf Wahlkreise aufgeteilt, in denen die Sitze im Verhältnis zu den abgegebenen Stimmen vergeben werden. Dabei werden erstmals Abgeordnete anstatt direkt vom Bürger indirekt über Listen gewählt. Die nicht vorhandene Möglichkeit des Wählers, direkt auf die Reihenfolge der Kandidaten Einfluss zu nehmen, sondern nur allgemein eine Partei und deren Liste wählen zu können, hat zu intensiven und kontroversen Diskussionen im britischen Parlament sowie in der Öffentlichkeit geführt.

Das Wahlrecht zum Unterhaus wurde trotz verschiedenster Vorschläge nicht reformiert. Labour und trotz der Wahlniederlagen auch die Konservativen waren nicht bereit, auf ein Wahlrecht zu verzichten, das ihnen bisher große Erfolge eingebracht hat. Insbesondere nach den Erfahrungen in Schottland und Wales, wo Labour durch das Wahlrecht in Koalitionen gezwungen wurde, sprach sich Tony Blair klar gegen eine Reform aus. ${ }^{30}$ Ein wichtiges Argument waren dabei die Erfahrungen in Deutschland, wo kleine Parteien mit fünf oder sechs Prozent Unterstützung in der Wählerschaft darüber bestimmen, wer die Regierung bildet. Der durchschnittlich sozialisierte Brite reagiert auf eine solche „Diktatur der Minderheit" und eine derartige „Interpretation“ des Wählerwillens in und durch Koalitionsverhandlungen mit völligem Unverständnis.

\subsection{Verhältnis zur Europäischen Union}

War die Europaskepsis in der britischen Öffentlichkeit auch beim Amtsantritt Blairs 1997 noch weit verbreitet, hatte sich zumindest die Situation innerhalb von Labour gewandelt. Mit Blair hatte Großbritannien einen Regierungschef, der im Gegensatz zu seinen Vorgängern - aus der Labour Party, aber vor allem im Vergleich zu den beiden vorhergehenden

29 Vgl. Bernd Becker, a.a.O. (Fn. 4), S. 66, S. 70; The Constitution Unit, http://www.ucl.ac.uk/ constitution-unit/publications/constitutional-update/elections-parties.html (Abruf am 13. November 2009).

30 Vgl. Bernd Becker, a.a.O. (Fn. 4), S. 223. 
konservativen Premierministern - erheblich europafreundlicher eingestellt war. An erster Stelle standen bei seiner Europapolitik die Interessen Großbritanniens. Er begriff die Europäische Union als Chance für Großbritannien und bezeichnete dabei die bisherige Politik als „Geschichte der verpassten Gelegenheiten“, weil Konservative und Labour die Realitäten der Einigung falsch eingeschätzt und somit den Interessen Großbritanniens geschadet hätten. ${ }^{31}$ Die unverzüglich nach dem Wahlsieg durchgeführte Entlassung der Bank of England in die faktische Unabhängigkeit erfüllte eine der wichtigsten Forderungen zur Ermöglichung eines Beitritts zur Europäischen Währungsunion und damit zum Euro. Im Vertrag von Amsterdam von 1997 stimmte Großbritannien einer Stärkung der Rechte der Europäischen Kommission und des Europäischen Parlamentes sowie einer gemeinsamen europäischen Außen-, Sicherheits-, Innen- und Rechtspolitik zu und akzeptierte damit eine Verringerung der Kompetenzen der Mitgliedstaaten. Im Vertrag von Nizza 2000 wurden nun auch unter Mitwirkung von Großbritannien institutionelle Reformen und die Charta der Grundrechte der EU ausgehandelt und verabschiedet. Außerdem unterzeichnete Großbritannien endlich die Europäische Sozialcharta, die von Margaret Thatcher noch als „Sozialismus durch die Hintertür" entschieden bekämpft worden war.

\subsection{Reform der Grundrechte}

Aufgrund der besonderen Struktur des Regierungssystems findet sich in Großbritannien keine verbindliche Festlegung von besonders geschützten Grundrechten. Die Einführung eines Grundrechtekataloges fordert automatisch die uneingeschränkte Parlamentssouveränität heraus, da diese von höherrangigem Recht bedroht würde. Der Sinn von Grundrechten ist es aber, höherrangig zu sein und gerade nicht von der einfachen Mehrheit im Parlament geändert werden zu können. Dieser Widerspruch ist an sich nicht aufösbar. Internationale Konventionen und Verträge zum Schutz der Menschenrechte werden folglich auch nicht automatisch geltendes (höherrangiges) Recht im Vereinigten Königreich, sondern müssen erst durch ein Gesetz des Unterhauses in gültiges Recht umgesetzt werden. Obwohl das Land seit langem Mitglied der UNO ist und der Europäischen Konvention zum Schutz der Menschenrechte beigetreten ist, ergibt sich der paradoxe Umstand, dass das Unterhaus weiterhin Gesetze erlassen kann, die diesen Konventionen widersprechen. Die britischen Gerichte dürfen sich auf sie aber nur dann berufen, wenn in dem zu verhandelnden Fall kein anders lautendes Gesetz des Unterhauses existiert. Erst unter Blair kam es zu einer pragmatischen Einführung von (teilweise) gesetzlich festgeschriebenen Grundrechten. Sie werden formal wie normales, jederzeit änderbares Recht behandelt, während dies aufgrund des gegenüber anderen Gesetzen erheblich größeren öffentlichen Interesses tatsächlich nur erschwert möglich ist.

Mit dem Human Rights Act von 1998, der am 2. Oktober 2000 in Kraft trat, wurde von der Labour-Regierung der Versuch gestartet, das „Unvereinbare zu vereinbaren“. Die Europäische Menschenrechtskonvention wurde in gültiges britisches Recht umgesetzt. Die

31 Vgl. ebenda, S. 292 ff.; Roger Morgan, Großbritannien und Europa, in: APuZ, B 18 (1997), S. 22 - 30, S. 30; Roland Sturm, a.a.O. (Fn. 25), S. 367; Jorgen Rasmussen, „What Kind of Vision is That?" British Public Attitudes Towards the European Community During the Thatcher Era, in: British Journal of Political Science, 27. Jg. (1997), H. 1, S. 111 - 115, S. 114 f. 
Regierung implementierte eine teilweise, aber nicht vollständige Bindung des Unterhauses an Grundrechte und gleichzeitig eine teilweise, aber nicht vollständige Kontrolle der Einhaltung der Grundrechte durch Gerichte. Vor der Verabschiedung eines Gesetzes im Unterhaus muss der zuständige Minister eine Stellungnahme abgeben, ob der Entwurf mit den Grundrechen vereinbar ist. Allerdings steht dem Unterhaus weiterhin die Möglichkeit offen, Gesetze zu erlassen, die dem Human Rights Act widersprechen. Dieser ermöglicht es den britischen Gerichten nicht, Gesetze aufzuheben, wenn sie die Grundrechte verletzen. Allerdings sind die Gerichte nun angehalten, bei der Anwendung und Bewertung aller existierenden und zukünftigen Gesetze zuerst den Human Rights Act und die Entscheidungen des Europäischen Gerichtshofes für Menschenrechte in Straßburg heranzuziehen. In der Praxis wird den Grundrechten somit ein Vorrang vor anderen Gesetzen eingeräumt.

\subsection{Reform des Amtes des Lordkanzlers}

Der Lord High Chancellor of Great Britain oder kurz: Lordkanzler symbolisierte in seiner Person die Machtfusion des Westminster-Modells. Er leitete die Sitzungen des Oberhauses und war in dieser Funktion Teil der Legislative. Dazu war er Mitglied im Kabinett und übernahm die Aufgaben eines Justizministers. Als Vorsitzender der Law Lords bekleidete er das Amt des Obersten Richters. Im Lordkanzler vereinigten sich also Exekutive, Legislative und Judikative. Im Zuge der Verfassungsreformen wurde auch diese Verkörperung des Westminster-Modells umgestaltet. Dies geschah vor allem, weil die Gewaltenfusion in diesem Amt immer weiter in die Kritik geraten war. Außerdem sollte dadurch die Unabhängigkeit der Gerichte gestärkt werden, um den Vorgaben der Europäischen Menschenrechtskonvention mehr Rechnung zu tragen. Daneben hatte sich die Zusammensetzung des Oberhauses geändert - ein Prozess, dessen Ende noch nicht absehbar ist. ${ }^{32}$ Zunächst plante die Regierung, den Lordkanzler und die Law Lords vollständig abzuschaffen. Dies stieß aber auf erbitterten Widerstand, nicht zuletzt, weil die Gewaltenfusion in diesem Amt immerhin viermal so lange Bestand hatte, wie es die Theorie der Gewaltenteilung von Montesquieu gibt. In Großbritannien, wo sich das Unterhaus zugunsten des Ziegenleders weigert, Gesetze auf Papier zu veröffentlichen, ist dieses Alter ein Argument von großem Gewicht.

Am 21. März 2005 wurde der Constitutional Reform Act 2005 von beiden Kammern verabschiedet, die ihn bereits seit Anfang 2004 beraten hatten. Der Lordkanzler ist nun nicht mehr Vorsitzender des Oberhauses. Es wurde die neue Position des Lord Speaker geschaffen, der vom Oberhaus selbst gewählt wird. Daneben wurde die Einrichtung eines Obersten Gerichtes beschlossen. In diesem Supreme Court sind die Law Lords die ersten Richter und behalten ihren Sitz im Oberhaus. Später soll eine Auswahlkommission eingesetzt werden, die neue Mitglieder des Gerichts auswählt, die dann auch nicht mehr Lords im Oberhaus sein sollen. Der Lordkanzler bleibt als Minister für Verfassungsfragen (De-

32 Ein gewichtiges Problem ergibt sich zum Beispiel dadurch, dass die Law Lords zwar Mitglieder des Oberhauses sind, aber sich nicht an den Sitzungen beteiligen. Sollte es zu einer Verkleinerung des Oberhauses oder zu einer teilweisen Volkswahl von Mitgliedern kommen, würden die Richter somit Plätze quasi sperren. 
partment for Constiutional Affairs. Justice, Rights and Democracy ${ }^{33}$ ) als Kabinettsmitglied erhalten und beaufsichtigt nun die Justiz, ohne selbst Richter zu sein. Die Aufgaben des Lordkanzlers als Oberster Richter hat jetzt der Lord Chief Justice übernommen.

\section{Ursachen und Folgen des Verfassungswandels}

Die Verfassungsreformen unter Tony Blair nach 1997 lassen sich nicht allein auf die lange Regierungszeit der Konservativen von 1979 bis 1997 zurückführen, die allerdings Umfang und Reichweite der Änderungen erklärt. Während der achtzehnjährigen Regierungszeit der Konservativen haben sich verschiedene Verfassungsprobleme, die durchaus schon vorher diskutiert wurden, zu einem Reformstau verdichtet. Zu nennen sind hierbei vor allem die immer stärker gewordenen Verzerrungen durch das Wahlsystem und die als rücksichtslos empfundene Politik von Margaret Thatcher, die den Bürgern das Gefühl gab, einem übermächtigen Staat hilflos ausgeliefert zu sein.

Der Verfassungswandel unter Blair hat die Handlungsspielräume des Unterhauses beziehungsweise des Premierministers und der ihn tragenden Mehrheit im Unterhaus entscheidend verändert. ${ }^{34}$ Im Oberhaus wurden die erblichen Sitze abgeschafft, so dass das Patronagepotential des Regierungschefs durch die Ernennung nicht-erblicher Mitglieder erheblich an Bedeutung gewonnen hat. Reformen mit dem Ziel, auch dem Unterhaus Einfluss einzuräumen, blieben hingegen erfolglos. Das Unterhaus selber hat vielmehr mit der Devolution in wichtigen Bereichen Gesetzgebungskompetenzen an regionale Vertretungen abgegeben und im Aufgabenbereich der Zentralbank Möglichkeiten verloren, die Wirtschaft zu beeinflussen. Im Verhältnis zur EU sowie beim Schutz der Grundrechte wurden ebenfalls umfassende Befugnisse abgetreten. Nicht einmal formal kann somit noch an der Doktrin der uneingeschränkten Parlamentssouveränität festgehalten werden. Auch ist das Unterhaus nicht länger die höchste Autorität bei der Interpretation der britischen „Verfassung“. So kann es real gegen den Widerstand der Bevölkerung die Devolution nicht zurücknehmen, auch wenn es juristisch das Recht dazu hätte.

Indirekt verbergen sich hinter der Devolution weitere Aspekte der Machtverschiebung: Die Erfahrungen mit den neu gewählten Volksvertretungen in Schottland und Wales zeigen, dass der Premierminister innerhalb seiner eigenen Partei an Einfluss verloren hat. So wurde Labour in Schottland nur durch das Wahlrecht in eine Koalition gezwungen. Der Einfluss des Regierungschefs endet nach der Devolution also an den Grenzen, die die regionalen Wahlergebnisse ziehen. Die Ereignisse nach der ersten Wahl in Wales vom 6. Mai 1999 machen diesen Einflussverlust besonders deutlich. Blair hatte seinen Vertrauten Alun

33 Vgl. die offizielle Homepage, http://www.justice.gov.uk/whatwedo/supremecourt.htm (Abruf am 13. November 2009).

34 Neben den hier aufgeführten Folgen des Verfassungswandels kam es zu weiteren nicht-intendierten Reformen, die durch die von Labour durchgeführten Verfassungsänderungen ausgelöst worden sind. Diese nicht-intendierten Reformen haben aber keinen Einfluss auf die Handlungsspielräume des Premierminister oder des Unterhauses (höchstens dahingehend, dass es einen öffentlichen Druck gibt, Stellung zu beziehen). Zu nennen sind hier zum Beispiel die West-Lothian-Question und das erfolglose Referendum vom 4. November 2004 zur Einführung gewählter Regionalversammlungen in den drei nordöstlichen Regionen Englands. Vgl. Merten Haring, a.a.O. (Fn. 10); André Kaiser, a.a.O. (Fn. 2). 
Michael gegen großen Widerstand in der eigenen Partei als Spitzenkandidaten durchgesetzt. Im Februar 2000 kam es während der Haushaltsberatungen zu einer schweren Krise, als ihm von den walisischen Abgeordneten vorgeworfen wurde, nicht ausreichend Geldmittel von London nach Wales zu holen und sich nicht genug für Wales einzusetzen. Er kam einem Misstrauensvotum durch seinen Rücktritt zuvor, und mit Rhodri Morgan wurde ein parteiinterner Gegner von Blair neuer Regierungschef. Im Oktober 2000 ging er mit den Liberaldemokraten eine formelle Koalition ein, weil ein Festhalten an herkömmlichen britischen Alleinregierungen trotz eines Verhältniswahlrechts und fehlender absoluter Mehrheit faktisch nicht durchzuhalten war. Zusammenfassend gesagt, hat der Premierminister aufgrund der Devolution parteiinterne Gegner, die sich auf eine eigene Machtbasis stützen und Politik gegen ihn machen können, gestärkt. Vor den Reformen wurden alle Posten vom Regierungschef in London vergeben. Dies hat sich mit der Abkehr vom reinen Mehrheitswahlrecht geändert, durch das die regionalen Parteien in Koalitionen gezwungen werden. Das Beispiel Gordon Brown zeigt, dass starke Gegenspieler innerhalb der eigenen Partei auch vor den Verfassungsreformen durchaus möglich waren - und vor allem in der Konservativen Partei gehört die Bildung von Flügeln zum guten Ton. Neu ist aber, dass sich Gegenspieler auf eine vom Premierminister unabhängige, institutionell abgesicherte und legitimierte Machtbasis stützen können; es gibt jetzt mehr Quellen der Souveränität als nur das Unterhaus.

Bei der Umsetzung der Devolution hatte Blair auf Referenden gesetzt, um die eigenen Reihen zu schließen. Gleichzeitig hatten die Konservativen in den Wahlkämpfen 1992 (erfolgreich) und 1997 die Furcht vor einem totalen Umbau des Staates durch Labour geschürt. Dagegen konnte Blair mit den geplanten Referenden argumentieren und versichern, dass die Reformen nicht gegen den Willen der Bürger geschehen würden.

Über die konkreten Volksabstimmungen zur Devolution hinaus hat sich unter Blair eine politische Konvention herausgebildet, deren Reichweite noch nicht beurteilt werden kann. Änderungen am institutionellen Gefüge stehen danach unter dem generellen Vorbehalt eines Referendums, um eine größere Zustimmung der Bevölkerung zu garantieren, als sie die durch das relative Mehrheitswahlrecht bestellte Regierung vertritt. Bei den Entwürfen zur europäischen Verfassung oder der Einführung des Euros forderte die Öffentlichkeit immer sofort ein Referendum, und keine Partei sprach sich mehr dagegen aus. Jüngstes Beispiel ist die Situation in Gibraltar: Obwohl Großbritannien und Spanien sich im Prinzip einig sind, das Gebiet wieder der Hoheit Spaniens zu unterstellen, hat ein von der britischen Regierung eigentlich nicht anerkanntes Referendum der Regionalregierung Gibraltars über diese Frage alle Verhandlungen gestoppt. Die Bürger sprachen sich fast einstimmig für den Verbleib bei Großbritannien aus, und niemand übergeht dieses Votum, obwohl es rechtlich keine Bedeutung hat.

Am Ende der Verfassungsreformen der Labour-Regierungen verfügt das Unterhaus formal weiterhin über alle Rechte bei institutionellen Veränderungen. Real muss aber jede Mehrheit im Unterhaus, die keine Referenden nach dem Vorbild Tony Blairs durchführt, damit rechnen, bei der nächsten Wahl abgestraft zu werden. Da die Volksabstimmungen und ihr Wortlaut maßgeblich vom Regierungschef beeinflusst werden, muss bilanziert werden, dass insbesondere diese Entwicklung tendenziell zu einer Entmachtung des Unterhauses und einer Stärkung des Premierministers geführt hat. 